\title{
The Impact of Genetic Variants on PTEN Molecular Functions and Cellular Phenotypes
}

\author{
Nicholas Hasle, ${ }^{1}$ Kenneth A. Matreyek, ${ }^{1}$ and Douglas M. Fowler ${ }^{1,2,3}$ \\ ${ }^{1}$ Department of Genome Sciences, University of Washington, Seattle, Washington 98195, USA \\ ${ }^{2}$ Department of Bioengineering, University of Washington, Seattle, Washington 98195, USA \\ ${ }^{3}$ Genetic Networks Program, CIFAR, Toronto, Ontario, M5G 1M1, Canada \\ Correspondence: dfowler@uw.edu
}

\begin{abstract}
Phosphatase and tensin homolog (PTEN) is a tumor suppressor that directly regulates a diverse array of cellular phenotypes, including growth, migration, morphology, and genome stability. How a single protein impacts so many important cellular processes remains a fascinating question. This question has been partially resolved by the characterization of a slew of missense variants that alter or eliminate PTEN's various molecular functions, including its enzymatic activity, subcellular localization, and posttranslational modifications. Here, we review what is known about how PTEN variants impact molecular function and, consequently, cellular phenotype. In particular, we highlight eight informative "sentinel variants" that abrogate distinct molecular functions of PTEN. We consider two published massively parallel assays of variant effect that measured the effect of thousands of PTEN variants on protein abundance and enzymatic activity. Finally, we discuss how characterization of clinically ascertained variants, establishment of clinical sequencing databases, and massively parallel assays of variant effect yield complementary datasets for dissecting PTEN's role in disease.
\end{abstract}

$\mathrm{P}_{\mathrm{is}}^{\mathrm{h}}$ hosphatase and tensin homolog (PTEN) is a tumor suppressor that affects cellular growth, morphology, and DNA repair (Box 1). PTEN is 403 amino acids in length and has two domains, an amino-terminal phosphatase domain and a carboxy-terminal "C2 domain" with homology to tensin, a cytoskeleton-associated protein. Following the carboxy-terminal domain, an approximately 50-residue unstructured region regulates PTEN activity. PTEN has molecular functions at the plasma membrane, cytoplasmic vesicles, and in the nucleus, including lipid phosphatase activity against phosphoinositol lipids; protein phosphatase activity against membrane-associated and nuclear pro- teins, and enigmatic nonenzymatic functions that primarily regulate nuclear protein activity (Box 1; Table 1).

Genetic variation in PTEN causes human disease. PTEN is either somatically mutated or deleted in $\sim 9 \%$ of all cancers. Germline PTEN variation causes PTEN hamartoma syndrome (PHTS), which is broadly characterized by macrocephaly, benign growths called hamartomas, and an increased risk of malignancy (Marsh et al. 1999; Yehia et al. 2019). Interestingly, many individuals with PHTS also have developmental delay and/or autism spectrum disorder, suggesting that PTEN also plays important roles in neurodevelopment (Butler et al. 2005). The

Editors: Charis Eng, Joanne Ngeow, and Vuk Stambolic

Additional Perspectives on The PTEN Family available at www.perspectivesinmedicine.org

Copyright (C) 2019 Cold Spring Harbor Laboratory Press; all rights reserved; doi: 10.1101/cshperspect.a036228

Cite this article as Cold Spring Harb Perspect Med 2019;9:a036228 
N. Hasle et al.

\section{BOX 1. PTEN MOLECULAR FUNCTIONS AND THE CELLULAR PHENOTYPES THEY IMPACT}

PTEN has both enzymatic and nonenzymatic molecular functions, acting as a lipid phosphatase, a protein phosphatase, and a protein scaffold. These molecular functions mediate PTEN's effects on cell growth, morphology, migration, genome stability, and DNA repair. PTEN's principal molecular function is as a phosphatase. The amino-terminal domain contains a HCXXGXXR phosphatase catalytic motif, which is capable of dephosphorylating both lipid and protein substrates (Myers et al. 1997). The primary lipid target of PTEN is phosphoinositide- $(3,4,5)$ phosphate (PIP3), an allosteric activator of the progrowth and prosurvival Akt/PKB pathway (Myers et al. 1998). PTEN's protein phosphatase activity shows broad target specificity, including (1) membrane associated proteins, such as focal adhesion kinase (FAK) (Tamura et al. 1998) and protein tyrosine kinase 6 (PTK6) (Wozniak et al. 2017); (2) nuclear proteins, such as cyclic AMP response element-binding protein (CREB) (Gu et al. 2011); and (3) mitotic spindle-associated proteins, such as EG5 (He et al. 2016).

PTEN also has nonenzymatic molecular functions that involve direct interaction with and modulation of downstream protein effectors in the nucleus. Two clear examples of such scaffolding activity have been reported. For example, PTEN's interaction with the anaphase-promoting complex, APC/C, increases its ubiquitination of various cell-cycle effectors (Song et al. 2011). Furthermore, PTEN interacts with RPA1 via its amino-terminal phosphatase domain and promotes RPA1 protein stability via recruitment of OTUB1 to collapsed replication forks (Wang et al. 2015). Finally, PTEN's carboxy-terminal unstructured region interacts with and inhibits PREX2, a guanineexchange factor that activates cell migration (Mense et al. 2015).

PTEN's diverse array of molecular targets and interactions is matched by its effects on many cellular phenotypes. PTEN knockout is associated with a number of cancer hallmarks, including uncontrolled cell growth, increased tissue invasion, and genome instability. The mechanisms behind each of these cellular phenotypes are multifaceted. For example, PTEN inhibits cell growth by decreasing Akt/PKB signaling (Myers et al. 1998), by suppressing CREB transcriptional activation (Gu et al. 2011), and by promoting APC/C complex activity (Song et al. 2011). It suppresses invasion by dephosphorylating FAK, PTK6, and perhaps an unidentified lipid substrate; and by interacting with PREX2 (Tamura et al. 1998; Tibarewal et al. 2012; Mense et al. 2015; Wozniak et al. 2017). Finally, PTEN promotes DNA repair by stabilizing RPA1 and inducing FANC-mediated DNA repair at collapsed replication forks, among other uncharacterized mechanisms (Bassi et al. 2013; Wang et al. 2015; Vuono et al. 2016).

relationships between PTEN sequence, molecular function, structure, impact on cellular phenotype, and these clinical outcomes are only partly understood. As such, it is challenging to predict the effect of most clinically observed PTEN variants on somatic cancer progression or on the outcomes of patients carrying germline PTEN variants.

Studies of PTEN missense variants have helped resolve the relationships between molecular function and cellular phenotypes, as the resulting PTEN mutant proteins can lack individual molecular functions. Such separation-offunction variants specifically alter or abrogate some protein function while preserving others. Comparing the molecular and cellular effects of separation-of-function variants can reveal how particular protein domains, surface pockets, and residues map to molecular functions and cellular phenotypes. Indeed, for the past 20 years, biologists have leveraged such missense variants to help dissect PTEN's functionality.

Here, we review the impact of missense variants on PTEN's molecular functions and cellular phenotypes. We identify eight separation-offunction “sentinel variants" that affect PTEN's enzymatic activities, subcellular localization, and posttranslational modifications. We also discuss what each variant has revealed about the connections between PTEN molecular functions and their effects on cellular phenotype. We discuss how multiplex assays of variant effects (MAVEs) are permitting the dissection of PTEN variant functionality in a comprehensive man- 
ner. Finally, we examine how clinical sequencing and variant characterization have shed light on the relationships between PTEN's molecular functions and human disease, and we highlight clinically observed missense variants that merit further study.

\section{PTEN VARIANTS THAT AFFECT ENZYMATIC ACTIVITY}

Shortly after PTEN's discovery as a tumor suppressor, variants affecting PTEN's phosphatase catalytic motif were noted in human cancers and in patients with PHTS (Li et al. 1997; Liaw et al. 1997; Marsh et al. 1999). These observations implied that PTEN's phosphatase activity is important for its tumor suppressor function. However, PTEN has many potential phosphatase substrates, including the lipid substrate phosphoinositide- $(3,4,5)$ phosphate (PIP3), which antagonizes the progrowth Akt/PKB pathway (Myers et al. 1998), as well as diverse protein substrates such as CREB (Gu et al. 2011), FAK (Tamura et al. 1998; Hu et al. 2014; Zhang et al. 2014), and PTK6 (Wozniak et al. 2017). To better understand which substrates are important for PTEN's tumor suppressive capability, PTEN mutants that abrogate specific catalytic functions were characterized (Myers et al. 1998; Davidson et al. 2010). Three such mutants are of particular interest: G129E, Y138L, and C124S. These mutants selectively abrogate PTEN lipid phosphatase activity, protein phosphatase activity, or both activities, respectively (Table 2).

Experiments with G129E, Y138L, and C124S revealed that PTEN's lipid phosphatase activity is critical for suppression of cell growth. This conclusion is based on three distinct assays for PTEN lipid phosphatase activity: in vitro activity against PIP3, in vivo assessment of PIP3 levels, and in vivo assessment of phospho-AKT/PKB levels. Wild-type PTEN and the Y138L mutant, which both possess lipid phosphatase activity in vitro, suppressed phospho-AKT/PKB levels and cellular growth in cell culture models (Davidson et al. 2010). However, the G129E and C124S mutants, which lack in vitro PIP3 phosphatase activity, did not suppress PIP3 levels in vivo or inhibit cell growth (Myers et al. 1998; Stambolic et al. 1998). Thus, PTEN hydrolysis of PIP3 leads to inhibition of the progrowth PI3K-AKT/PKB pathway because PIP3 is an allosteric activator of PI3K. Importantly, subsequent work revealed that stable mutants without lipid phosphatase activity can also dimerize with and suppress the lipid phosphatase activity of wild-type alleles in a dominant negative manner (Papa et al. 2014).

Although loss of PTEN protein phosphatase activity does not affect cell growth, further studies of cells expressing the G129E mutant revealed that it can regulate cellular morphology and migration. For example, expression of this protein-phosphatase competent mutant inhib-

Table 1. PTEN molecular functions and impacted cellular phenotypes

\begin{tabular}{llll}
\hline Molecular function & Molecular target(s) & \multicolumn{1}{c}{ Cellular phenotype } & \multicolumn{1}{c}{ References } \\
\hline Lipid phosphatase & PIP3 & $\downarrow$ Cell growth and survival & Myers et al. 1998; Davidson \\
& & $\downarrow$ Migration & et al. 2010; Tibarewal et al. \\
Protein phosphatase & PTK6, FAK & $\downarrow$ Cell spreading & 2012 \\
& & $\downarrow$ Migration & Tamura et al. 1998; Wozniak \\
& CREB & $\downarrow$ Cell spreading & et al. 2017 \\
& & $\downarrow$ Transcription of growth- & Gu et al. 2011 \\
& related genes & \\
FANC proteins? & $\uparrow$ DNA repair & Bassi et al. 2013; Vuono et al. \\
Nonenzymatic & APC/C & $\uparrow$ Delay of G1-S transition & Song et al. 2011 \\
(scaffolding) & PREX2 & $\downarrow$ Migration & Mense et al. 2015 \\
& RPA1, OTUB1 & $\uparrow$ RPA1-mediated resolution & Wang et al. 2015 \\
& & of stalled replication forks & \\
\hline
\end{tabular}


N. Hasle et al.

Table 2. Separation of enzymatic function variants

\begin{tabular}{|c|c|c|c|c|c|c|c|}
\hline Variant & $\begin{array}{c}\text { Lipid } \\
\text { phosphatase } \\
\text { activity in } \\
\text { vitro }\end{array}$ & $\begin{array}{c}\text { PKB } \\
\text { suppression } \\
\text { in vivo }\end{array}$ & $\begin{array}{c}\text { Protein } \\
\text { phosphatase } \\
\text { activity }\end{array}$ & $\begin{array}{c}\text { Cell } \\
\text { growth } \\
\text { inhibition }\end{array}$ & $\begin{array}{c}\text { Migration } \\
\text { suppression }\end{array}$ & $\begin{array}{l}\text { DNA } \\
\text { repair }^{\text {a }}\end{array}$ & References \\
\hline Wild-type & + & + & + & + & + & + & $\begin{array}{l}\text { Myers et al. 1998; } \\
\text { Gildea et al. } \\
\text { 2004; Davidson } \\
\text { et al. 2010; Bassi } \\
\text { et al. 2013; } \\
\text { Vuono et al. } \\
2016\end{array}$ \\
\hline Y138L $L^{b}$ & + & + & - & + & $+1-$ & - & $\begin{array}{l}\text { Davidson et al. } \\
\text { 2010; Tibarewal } \\
\text { et al. 2012; } \\
\text { Vuono et al. } \\
2016\end{array}$ \\
\hline G129E & - & - & + & - & $+/-$ & + & $\begin{array}{l}\text { Myers et al. 1998; } \\
\text { Gildea et al. } \\
\text { 2004; Davidson } \\
\text { et al. 2010; Bassi } \\
\text { et al. 2013; } \\
\text { Vuono et al. } \\
2016\end{array}$ \\
\hline $\mathrm{C} 124 \mathrm{~S}^{\mathrm{b}}$ & - & - & - & - & $+1-$ & - & $\begin{array}{l}\text { Myers et al. 1998; } \\
\text { Gildea et al. } \\
\text { 2004; Davidson } \\
\text { et al. 2010; Bassi } \\
\text { et al. 2013; } \\
\text { Vuono et al. } \\
2016\end{array}$ \\
\hline Y138C & + & + & - & + & NS & NS & $\begin{array}{l}\text { Tibarewal et al. } \\
2012\end{array}$ \\
\hline M134L & - & - & + & - & NS & NS & $\begin{array}{r}\text { Myers et al. } 1998 ; \\
\text { Han et al. } 2000\end{array}$ \\
\hline
\end{tabular}

Included are thermodynamically stable PTEN mutants for which both lipid and protein phosphatase activities have been measured. Wild-type, Y138L, G129E, and C124S are commonly used in PTEN studies.

NS, not studied.

${ }^{\mathrm{a} D N A}$ repair capabilities as reported by Bassi et al. (2013) and Vuono et al. (2016).

bentinel variant.

ited glioblastoma cell line spreading on fibronectin (Tamura et al. 1998) and inhibited the migration and invasion of various cell lines (Gildea et al. 2004; Davidson et al. 2010; Mense et al. 2015). Mechanistically, these effects appear to be mediated by dephosphorylation and consequent inactivation of FAK and PTK6, which are kinases implicated in focal adhesion formation and invasion (Tamura et al. 1998; Zhang et al. 2014; Wozniak et al. 2017). Because G129E lacks lipid phosphatase activity, these studies suggested that PTEN's protein phosphatase activity inhibits cell morphology and migration.

However, studies of the C124S and Y138L variants reveal that PTEN's role in controlling invasion is complex, context-dependent, and not solely dependent on its protein phosphatase activity. For example, the C124S and Y138L mutants partially suppressed invasion of breast cancer and glioblastoma cell lines, respectively ( $\mathrm{Da}$ - 
vidson et al. 2010; Tibarewal et al. 2012; Mense et al. 2015). Furthermore, Y138L prevented the spread of a glioblastoma cell line grown on collagen IV to a greater extent than G129E, indicating that the lipid phosphatase activity is more important than the protein phosphatase activity in this context (Davidson et al. 2010). These results suggest that the lipid phosphatase activity is capable of impacting the morphology and migration, perhaps by modulation of PIP3 gradients or a signaling pathway that is activated by PIP3 or a related lipid (Davidson et al. 2010). Interestingly, the PTEN C124S mutant also impacted breast cancer cell line migration via interaction with and inactivation of PREX2 (Mense et al. 2015). More experiments are required to fully understand the relative importance and context-dependence of the protein and lipid phosphatase activities in enacting changes to cellular shape and invasion.

Studies of PTEN variants have found that PTEN's protein phosphatase function also promotes DNA repair. For example, enzymatically dead C124S was unable to induce a BRCA1-, 53BP1-, and RAD51-associated DNA damage response and, consequently, did not promote repair of dsDNA breaks in a cell culture model. However, the protein phosphatase competent G129E mutant could still do so (Bassi et al. 2013). Similarly, the protein phosphatase null Y138L mutant could not recruit FANC proteins to interstrand cross-links to enact DNA repair in murine fibroblasts and HeLa cells, but the G129E mutant could (Vuono et al. 2016). Thus, PTEN's protein phosphatase function appears to coordinate the activity of many DNA repair-related proteins, including FANC proteins, BRCA1/2, and 53BP1, although the mechanism remains a mystery.

Some PTEN nuclear functions do not require any enzymatic activity at all. For example, C124S, which is enzymatically inactive, promoted rescue of stalled replication forks via interaction with RPA1 in a colon cancer cell line (Wang et al. 2015). This "scaffolding function" was also noted by Song and colleagues, who reported that $\mathrm{C} 124 \mathrm{~S}$ was still able to delay the $\mathrm{G}_{1}-\mathrm{S}$ transition by interacting with APC/C in murine fibroblasts and cancer cell lines (Song et al. 2011). Thus, PTEN appears to function as a protein phosphatase and a protein scaffold as it fulfills its diverse nuclear roles. The role of the lipid phosphatase in the nucleus merits further study, perhaps by assaying the Y138L variant for its effects on PTEN's nuclear functions.

The studies of PTEN function highlighted here mostly compared the effects of one or two PTEN variants to wild-type. To better understand how enzyme activity affects function, future studies might benefit from including at least three variants: C124S (enzymatically null), G129E (lipid-phosphatase null), and Y138L (protein-phosphatase null). We recommend inclusion of these three variants because PTENrelated cellular phenotypes can depend on just one activity (e.g., AKT/PKB-mediated cell growth), neither (e.g., G1-S transition), or both (e.g., cell migration; Table 2). One area of particular confusion is cellular migration and invasion, in which different conclusions have been drawn regarding the relative importance of each phosphatase activity (Gildea et al. 2004; Davidson et al. 2010; Tibarewal et al. 2012; Mense et al. 2015). These conflicts may result from differences in cell line or assay conditions, and additional studies that investigate the contextdependence of PTEN enzyme activity on cell migration are needed.

\section{PTEN VARIANTS THAT AFFECT SUBCELLULAR LOCALIZATION}

The subcellular localization of PTEN controls its access to lipid and protein substrates. PIP3 and at least two of PTEN's protein phosphatase targets, FAK and PTK6, are localized to the plasma membrane (Schaller et al. 1992; Ie Kim and Lee 2009). Furthermore, PTEN interacts with and dephosphorylates protein targets in the nucleus. Reflecting the importance of PTEN localization on its molecular functions, investigators have found and characterized variants that preclude PTEN localization to the plasma membrane and nuclear compartments. These variants have helped reveal the relationships between PTEN's subcellular distribution and its impact on cellular phenotypes. 
N. Hasle et al.

PTEN associates spontaneously with membranes without requiring protein-protein interactions or posttranslational modification with lipid moieties. The crystal structure of PTEN revealed a number of hydrophobic and basic regions that are likely involved in membrane binding (Fig. 1). These regions are located on the active-site face of PTEN, which presumably must interact directly with the membrane to access its lipid substrate. The membrane binding regions include the CBR3 loop (positions 263269), the c $\alpha 2$ element (positions 327-335), the WPD loop of the active site (positions 90-93), and the TI loop (positions 161-164) (Fig. 1; Lee et al. 1999). Subsequent studies based on the structure showed that mutations in these regions led to decreased membrane binding in vitro, in a slime mold model, and in cell culture (Das et al. 2003; Nguyen et al. 2014b, 2015).

Additional mutagenesis studies revealed new residues necessary for lipid substrate binding and verified that loss of PTEN membrane localization results in loss-of-growth inhibition. For example, mutation of four amino-terminal basic residues to alanine resulted in a 76-fold decrease in PTEN's affinity for lipid vesicles in vitro and decreased plasma membrane localization in cell

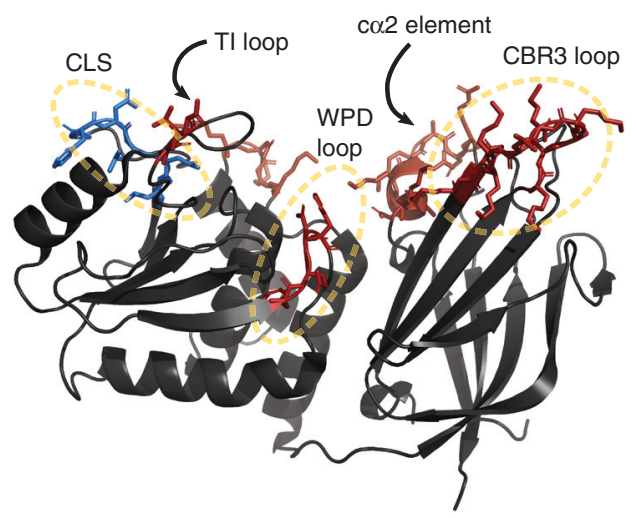

Figure 1. PTEN residues that affect subcellular localization. PTEN (PDB: 1D5R) interfaces with the membrane at the top of the structure. Residues colored in red are necessary for membrane binding and localization. Mutation of the blue residues result in in creased nuclear localization. CLS, cytoplasmic localization sequence (Denning et al. 2007). (Structure from Lee et al. 1999.) culture (Das et al. 2003). Two individual mutations affecting these positions, R14A and R15A, were subsequently found to decrease PTEN membrane localization in cell culture and severely reduce its in vitro lipid phosphatase activity (Han et al. 2000; Nguyen 2014b). Interestingly, some cancer-associated mutations in the amino terminus (e.g., S10N) and the phosphatase domain (e.g., G20E, L42R, F90S) yielded competent PIP3 phosphatases in vitro but compromised membrane localization and Akt/PKB pathway inhibition in cells (Table 3; Han et al. 2000; Nguyen et al. 2015). This suggests that the in vitro PIP3 phosphatase assay will not reflect phosphatase activity in vivo if the PTEN mutant cannot localize to the membrane.

Membrane-binding defective PTEN mutants also lose access to membrane-localized protein substrates. Nguyen and colleagues showed that the cancer-associated, lipid-binding defective S10N, G20E, L42R, and F90S mutants also lost the ability to suppress breast cancer cell transwell migration (Nguyen et al. 2015). Two of PTEN's protein phosphatase targets thought to be involved in cell migration, FAK and PTK6, are membrane localized, potentially explaining this observation (Schaller et al. 1992; Ie Kim and Lee 2009). Notably, S10N, G20E, and F90S (but not L42R) also caused increased nuclear localization in a slime-mold model (Nguyen et al. 2015). However, the effect of these mutants on DNA repair and chromosome stability has not been evaluated and merits further study.

In addition to the numerous regions that drive PTEN membrane localization, there appears to be a region important for maintaining wild-type levels of PTEN in the cytoplasm. Mutants of positions 19-25 were found in tumors and result in constitutive PTEN nuclear localization in HEK293Ts. One of these mutants, F21A, showed wild-type-like lipid phosphatase activity in vitro, but lost almost all lipid phosphatase activity in vivo, as measured by AKT/PKB signaling and glioblastoma cell line growth (Denning et al. 2007). Importantly, how these variants cause nuclear localization is unknown; positions 19-25 may constitute a noncanonical nuclear export sequence, or the mechanism might in- 
Table 3. Separation of localization function variants

\begin{tabular}{|c|c|c|c|c|c|c|}
\hline Variant & $\begin{array}{c}\text { Lipid } \\
\text { phosphatase } \\
\text { activity in vitro }\end{array}$ & $\begin{array}{c}\text { PKB } \\
\text { suppression } \\
\text { in vivo }\end{array}$ & $\begin{array}{l}\text { Membrane } \\
\text { localization }\end{array}$ & $\begin{array}{l}\text { Cytoplasmic } \\
\text { localization }\end{array}$ & $\begin{array}{c}\text { Nuclear } \\
\text { localization }\end{array}$ & References \\
\hline $\begin{array}{l}\text { Wild- } \\
\text { type }\end{array}$ & + & + & + & + & + & $\begin{array}{r}\text { Myers et al. 1998; } \\
\text { Das et al. } 2003\end{array}$ \\
\hline $\mathrm{L}_{4} 2 \mathrm{R}^{\mathrm{a}}$ & + & - & - & + & + & $\begin{array}{l}\text { Han et al. 2000; } \\
\text { Nguyen et al. } \\
2015\end{array}$ \\
\hline $\mathrm{F} 21 \mathrm{~A}^{\mathrm{a}}$ & + & - & - & - & ++ & Denning et al. 2007 \\
\hline S10N & + & - & - & + & ++ & $\begin{array}{l}\text { Han et al. 2000; } \\
\text { Nguyen et al. } \\
2015\end{array}$ \\
\hline G20E & + & - & - & + & ++ & $\begin{array}{l}\text { Han et al. 2000; } \\
\text { Nguyen et al. } \\
2015\end{array}$ \\
\hline F90S & NS & - & - & + & ++ & $\begin{array}{l}\text { Han et al. 2000; } \\
\text { Nguyen et al. } \\
2015\end{array}$ \\
\hline $\mathrm{R} 14 \mathrm{~A}$ & NS & NS & - & + & NS & Nguyen et al. 2015 \\
\hline $\mathrm{R} 15 \mathrm{~A}$ & NS & NS & - & + & NS & Nguyen et al. 2015 \\
\hline
\end{tabular}

volve increased ubiquitination, sumoylation, and/ or tyrosine phosphorylation. Furthermore, the effects of these nuclear-localized mutants on cellular migration and DNA repair is unclear.

For future studies of PTEN's effects on cell biology, we recommend including the cancerassociated, membrane-binding defective L42R and nuclear-localized F21A mutants. These mutants appear to be stable, show lipid phosphatase activity in vitro, and are not known to affect posttranslational modification. Thus, these separation-of-function variants appear to modulate PTEN's localization rather than its catalytic activity. As such, the downstream effects of PTEN's dissociation from the membrane and constitutive localization to the nucleus can be studied by comparing the phenotypes of cells expressing the L42R and F21A sentinel variants to wild-type PTEN.

\section{PTEN VARIANTS THAT AFFECT POSTTRANSLATIONAL MODIFICATIONS}

The L42R and F21A variants discussed above appear to affect localization of PTEN in a constitutive manner. However, PTEN is also subject to dynamic regulation of its localization by posttranslational modifications (PTMs), including serine/threonine phosphorylation, tyrosine phosphorylation, ubiquitination, and sumoylation (Fig. 2). These PTMs regulate PTEN stability and activity in addition to localization, and they may perform additional roles such as regulating protein-protein interactions. PTEN missense variants that preclude posttranslational modification have helped reveal the molecular and cellular consequences of PTEN regulation by PTMs (Table 4).

PTEN's activity, membrane localization, and stability are all regulated by phosphorylation of its carboxy-terminal tail. Glycogen synthase kinase $3 \beta$ and casein kinase were found to be responsible for these phosphorylation events (Torres and Pulido 2001; Al-Khouri et al. 2005), which in cell culture models resulted in a closed conformational state that could not bind the membrane, showed increased stability, and did not have lipid phosphatase activity (Vazquez et al. 2000; Das et al. 2003; Odriozola et al. 2007; Rahdar et al. 2009). Mutation of two of these phosphorylated residues to alanine (T366A/S370A) increased PTEN-mediated 
N. Hasle et al.

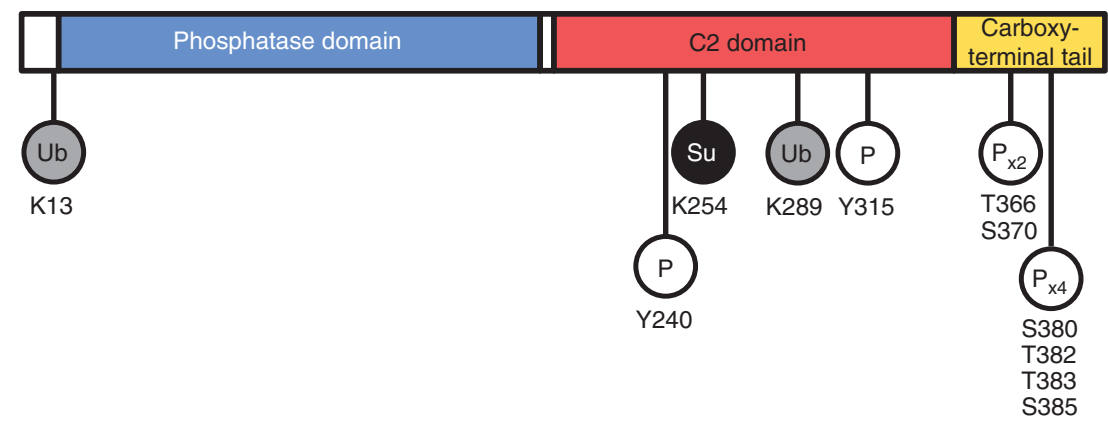

Figure 2. PTEN posttranslational modifications. Ub, ubiquitination; Su, sumoylation; $\mathrm{P}$, phosphorylation; Px2, Px4, residues that are cophosphorylated (Vazquez et al. 2000; Al-Khouri et al. 2005).

inhibition of AKT/PKB signaling in Jurkat cells, presumably by preventing this inactive conformational state (Al-Khouri et al. 2005). Concordantly, a second set of phosphorylation sites between residues 380 and 385 was identified, and mutation of four serine and threonine residues within this region to alanine (the "A4" variant) increased plasma membrane localization, increased in vivo lipid phosphatase activity, and reduced steady state PTEN abundance in various cell lines (Vazquez et al. 2000; Das et al. 2003; Rahdar et al. 2009; Nguyen et al. 2014b). Interestingly, PTEN may dephosphorylate itself at position 383 , opening its conformation and permitting its interaction with and inhibition of invasion-promoting PREX2 (Raftopoulou et al. 2004; Zhang et al. 2012; Mense et al. 2015).

PTEN is also regulated by tyrosine phosphorylation of its C2 domain, where both SRC- and PDGFR-family kinases phosphorylate PTEN on residues Y240 and Y315 (Koul et al. 2002; Nathanson et al. 2012). Y240F or Y315F mutants isolated from U251 glioma cells showed lower lipid phosphatase activity in vitro, suggesting that tyrosine phosphorylation is required for wild-type activity (Koul et al. 2002). However, loss of Y240 phosphorylation comes at a cost for cancer cells: this PTM was required for PTEN's role in DNA damage repair, and cells expressing Y240F were more sensitive to DNA damaging agents than their wild-type counterparts (Ma et al. 2019). Consistent with these findings, the majority of nuclear-localized
PTEN, which carries out PTEN's DNA repair promoting functions, were phosphorylated at Y240. Furthermore, ionizing radiation increased both the degree of PTEN Y240 phosphorylation and the amount of nuclear PTEN in glioma cell lines (Ma et al. 2019). These studies suggested that Y240/Y315 phosphorylation of PTEN is important in shifting its localization and activity from the cytoplasm to the nucleus.

Like phosphorylation, PTEN sumoylation has been implicated in regulation of PTEN localization, with critical consequences for PTEN activity at the plasma membrane and in the nucleus. PTEN was found to be sumoylated on residues K254 and K266, and mutation of these residues to sumoylation-incompetent arginine decreased membrane localization, decreased lipid phosphatase activity, and increased anchorage-dependent growth of an osteosarcoma line, compared with wild-type PTEN (Huang et al. 2012). Indeed, molecular dynamics simulations suggested that sumoylation improves membrane localization via SUMO1-mediated electrostatic interactions with the lipid bilayer (Huang et al. 2012). The K254R mutant has additional defects: decreased nuclear localization in HEK293T cells and lower DNA repair induction after DNA damage in various cell lines, compared with wild-type (Bassi et al. 2013; Vuono et al. 2016). Like tyrosine phosphorylation, loss of sumoylation can result in both lipid-phosphatase and DNA repair defects.

Ubiquitination is a third PTM that regulates PTEN localization and function. Ubiquitinated 

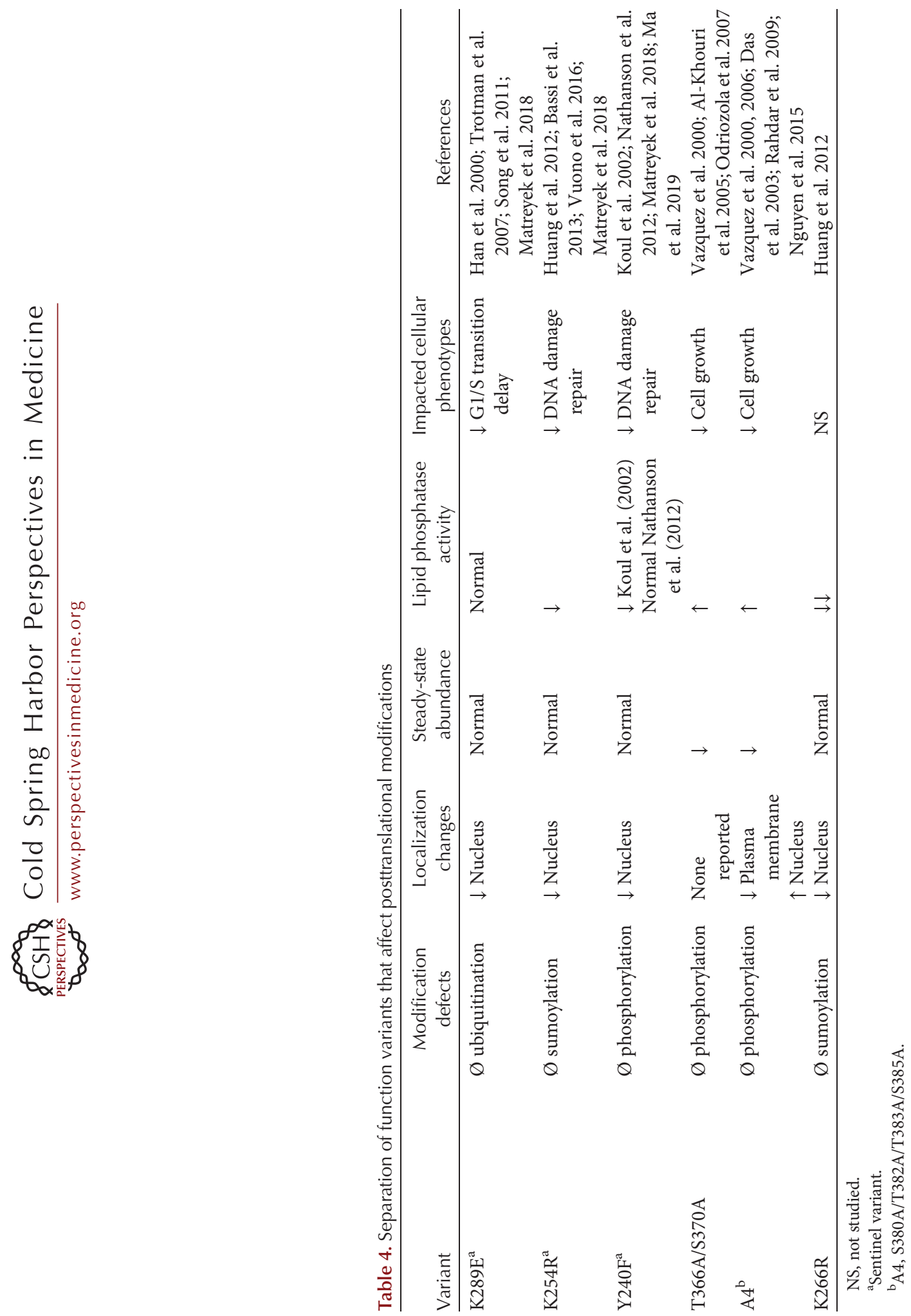
N. Hasle et al.

PTEN was discovered after gastrointestinal polyp biopsy of a PHTS patient carrying a germline K289E variant showed loss of nuclear PTEN. Characterization of this residue revealed that it is ubiquitinated by NEDD4-1, a membrane-localized ubiquitin ligase, and that loss of monoubiquitination in K289E resulted decreased of PTEN nuclear import (Trotman et al. 2007). Residue K13 was also found to be ubiquitinated and excluded from the nucleus in 293 cells if mutated to glutamate (Trotman et al. 2007; Wang et al. 2007). Furthermore, both mutants had a decreased capacity to delay the $\mathrm{G}_{1}-\mathrm{S}$ transition in PC-3 cells under normal growth conditions caused by an inability to interact with the nucleus-localized, tumor-suppressive APC/C complex (Song et al. 2011). NEDD4-1 could also polyubiquitinate PTEN at K289 and K13, and perhaps other sites, which led to protein degradation (Trotman et al. 2007). Polyubiquitination occurs less frequently than monoubiquitination for wild-type PTEN, but increased concentrations of NEDD4-1 or PTEN mutants missing the carboxy-terminal tail increased the degree of NEDD4-1 polyubiquitination and led to PTEN degradation (Wang et al. 2007). Thus, like tyrosine phosphorylation and sumoylation, ubiquitination affects the nuclear functions of PTEN. Additionally, ubiquitination is an important determinant of PTEN stability.

In conclusion, mutagenesis studies have revealed that PTEN PTMs can change the localization, enzymatic targets, or stability of PTEN, either individually or simultaneously. For example, loss of serine/threonine phosphorylation at residues 380-385 in the A4 variant decreases stability, increases membrane localization, and increases lipid phosphatase activity of PTEN. One outstanding question is how various PTMs cross talk with each other, particularly in relation to PTEN's nuclear localization, interaction with APC/C, and induction of DNA repair. Y240F (tyrosine phosphorylation-deficient), K254R (sumoylation-deficient), and K289E (ubiquitination-deficient) all appear to result in loss of nuclear PTEN. Is this because all three modified residues are necessary for nuclear localization? If so, are all nuclear functions of PTEN defective in each of these mutants?
Furthermore, the protein phosphatase activity and the effects of these mutants on migration have not been tested. More studies involving these three mutants will be required to better understand the relationships between PTEN PTMs, PTEN-induced DNA repair, and PTEN-APC/C interactions.

\section{USING HIGH-THROUGHPUT MUTAGENESIS TO CHARACTERIZE PTEN VARIANTS}

The traditional biochemical and cellular experiments described above have each focused on a handful of PTEN variants for a subset of activities. Unfortunately, assay conditions for these one-at-a-time approaches often differ between laboratories, which restricts the ability to compare variants directly, and performing them on more than several variants is laborious. MAVEs are powerful tools for comprehensively determining how changes to protein sequence affect protein function, which can be correlated with cell and disease phenotypes (Gasperini et al. 2016; Starita et al. 2017). MAVEs enable the simultaneous assessment of thousands of genetic variants in a single experiment. Recently, MAVEs were used to assess two complementary properties of PTEN: lipid phosphatase function within yeast (Mighell et al. 2018) and intracellular abundance in human-derived cell lines (Matreyek et al. 2018). By testing thousands of possible PTEN missense variants, these studies yielded new insights into the relationship between PTEN's sequence, structure, and function.

Mighell et al. adapted a humanized yeast model to assess PTEN lipid phosphatase activity at high throughput (Mighell et al. 2018). Expression of the human PI3K catalytic subunit p110 inhibits yeast cell proliferation, which can be rescued by the phosphatase activity of human PTEN (Rodríguez-Escudero et al. 2005). This assay was previously used to functionally characterize dozens of PTEN variants (Andrés-Pons et al. 2007; Rodríguez-Escudero et al. 2011). Mighell et al. incorporated a site-saturation mutagenesis library of PTEN into yeast and used high-throughput sequencing to measure the 
growth effects of each variant in the library in parallel. Of the 6564 missense variants that were scored with high confidence, 1789 variants showed activities below the range of scores observed for synonymous variants. Of these, 1249 had extremely low activities similar nonsense variants, with the remaining 540 variants showing an intermediate phenotype.

We developed variant abundance by massively parallel sequencing, or VAMP-seq (Matreyek et al. 2018), by fusing a library of PTEN single missense variants to EGFP. We used this assay to assess how each fused PTEN mutant altered EGFP levels and thus PTEN abundance. We assessed 4112 single-amino-acid mutants of PTEN, identifying 1138 mutants with clear loss of intracellular abundance. Thermodynamic stability appeared to be a major correlate for PTEN intracellular abundance, as a large subset of loss-of-abundance variants perturbed hydrophobic residues present within the core regions of each domain or at residues making hydrogen bonds in the PTEN crystal structure.

The results of these MAVEs are consistent with previous literature. For example, many variants altering PTEN's catalytic motif caused loss of lipid phosphatase activity in the yeast functional assay, but no change in steady state abundance. Variants abrogating serine/threonine phosphorylated sites in the carboxy-terminal tail, such as S385A, showed increased activity and reduced abundance, as expected. Nonetheless, these MAVEs also come with some caveats; for example, the K254R and K266R sumoylation-defective variants do not show reduced lipid phosphatase activity in the yeast assay, as would be expected. This may be because of the lack of a PTEN sumoylase ortholog in yeast.

MAVEs provide a comprehensive and nuanced understanding of how PTEN variants affect its molecular functions. For example, not all changes to the catalytic loop completely abrogated lipid phosphatase function, as computational approaches might predict. Furthermore, there were many active site mutants that showed normal abundance yet were completely inactive; these may represent dominant negatives that act in a similar manner to C124S and G129E (Papa et al. 2014). As more PTEN MAVEs are com- pleted, we expect that additional subtleties regarding PTEN's sequence-structure-function relationships will come to light.

\section{FUNCTIONAL IMPACTS OF CLINICALLY OBSERVED PTEN VARIANTS}

The analysis of clinical samples containing PTEN variants has helped elucidate the molecular and cellular mechanisms underlying PTENrelated disease. For example, when PTEN was discovered, variants of its catalytic motif sequence (e.g., G129R, M134L) were noted in diverse cancer types (Li et al. 1997; Liaw et al. 1997) Secondly, comparison of the germline variants associated with severe versus mild PHTS revealed that severe variants are often stable and catalytically inactive, whereas mild variants retain partial activity (Rodríguez-Escudero et al. 2011; Spinelli et al. 2015; Leslie and Longy 2016). Finally, Trotman et al. (2007) observed decreased PTEN nuclear localization after loss of heterozygosity in hamartomatous polyps in a patient with PHTS, which led to the discovery of the K289 ubiquitination site. These studies show that examination of uncharacterized patient variants has been and will continue to be vital for furthering our understanding of PTEN biology.

The application of exome and genome sequencing to human cancer and the diagnosis of genetic disorders is yielding a plethora of new clinical variant information. cBioPortal and ClinVar are databases that catalog somatic variants in cancer and germline variants in patients with PHTS, respectively (Cerami et al. 2012; Landrum et al. 2018; Mester et al. 2018). The relative prevalence of somatic PTEN variants in tumors and germline variants in patients with PHTS in these databases offer clues to regions of PTEN that have important functions. Indeed, $27 \%$ of all PTEN missense variants reported in the cBioPortal MSK-IMPACT cohort (Schrader et al. 2016) and 18\% of PTEN variants in ClinVar are located in the phosphatase catalytic motif, which constitutes $2 \%$ of the PTEN's length. Furthermore, the carboxy-terminal tail comprises $13 \%$ of PTEN's length, but only $1.3 \%$ of the missense variants reported in cancer genomes are in the tail, consistent with the tail's 
N. Hasle et al.

role in inhibiting PTEN access to PIP3 substrate at the plasma membrane (Vazquez et al. 2000; Das et al. 2003; Rahdar et al. 2009; Nguyen et al. 2014b).

Despite the use of single-variant studies that arise from clinical observation, most clinically reported variants have little functional data. Variants altering two positions, R130 and R173, show particularly high prevalence in clinical sequencing databases and have yet to be fully characterized. Arginine 130 is located within PTEN's HCXXGXXR catalytic motif and the MSK-IMPACT data shows that it comprises a staggering $65 \%$ of all missense variants in this region (Schrader et al. 2016). R130 variants are fairly stable (Spinelli et al. 2015; Matreyek et al. 2018), lack lipid phosphatase activity (Han et al. 2000; Mighell et al. 2018), and can exert dominant negative effects (Papa et al. 2014), just like G129E and C124S variants. However, these similarities do not explain why R130 variants are so much more common than $\mathrm{C} 124$ or G129 variants in cancer. Rather, it is likely that CpG dinucleotides, which constitute the first two dinucleotides of the R130 codon, are subject to higher mutation rates resulting from deamination. Given their high prevalence in cancers, additional work is needed to characterize the functional attributes and cellular consequences of R130 variants.

The PTEN codon encoding R173 is similarly susceptible to deamination mutations; such mutations constitute $8 \%$ of all somatic PTEN missense variants in cancer (Schrader et al. 2016) and have been repeatedly observed in patients with PTEN hamartoma tumor syndrome. The resulting PTEN mutants have reduced but not absent lipid phosphatase activity in Mighell and colleagues' yeast-based functional assay, suggesting that they have altered function. In the PTEN crystal structure, R173 is located in a helix with the TI-loop, an important determinant of phosphatase active site structure. This helix, and R173 in particular, also interacts with the C2 domain (Lee et al. 1999). Thus, the crystal structure suggests that R173 variants may affect the structure of the active site and/or interactions between the phosphatase and C2 domains. Indeed, a structural analysis of disease-associated residues suggested that $\mathrm{R} 173$ forms part of an allosteric network that dictates PTEN conformation (Smith et al. 2019). However, specific functional data on R173 mutants, including their protein phosphatase activities, localization, and effects on DNA repair, are lacking.

Before the publication of PTEN-specific MAVEs, the biochemical consequences of most clinically ascertained variants-not just those affecting R130 and R173-in PTEN were unknown. Molecular functional data represents a critical evidence type for the clinical interpretation of variants and can help classify clinically ascertained variants as benign or pathogenic (Richards et al. 2015; Mester et al. 2018). We reported that PHTS-related PTEN pathogenic germline variants in ClinVar were enriched in low-abundance variants, with $>80 \%$ of scored pathogenic variants being low or possibly low in abundance (Matreyek et al. 2018). Similarly, Mighell et al. (2018) found that $60 \%$ of known pathogenic PTEN variants had enzyme activity scores below the upper fifth percentile of the nonsense variant score distribution, corresponding to a positive predictive value of $98 \%$. They also found that germline variants associated with autism spectrum disorder had hypomorphic activity scores, supporting a previous hypothesis linking PTEN phosphatase activity with phenotype (Leslie and Longy 2016).

Given the success of these studies in predicting pathogenicity, the ClinGen PTEN expert panel has provided guidelines for how to incorporate MAVE-generated data into clinical interpretation of variants (Mester et al. 2018). As PTEN's lipid phosphatase activity appears to be most closely correlated with tumorigenesis, they recommend that functional assays measuring this molecular function, such as the yeast assay published by Mighell et al., provide the strongest evidence for pathogenicity. Examination of other biochemical deficits, such as loss of protein phosphatase activity, decreased abundance, or change in subcellular distribution, is also recommended to help determine whether or not a PTEN variant is pathogenic.

Although the two published MAVEs of PTEN have documented accuracy for classifying clinically ascertained germline PTEN variants, 

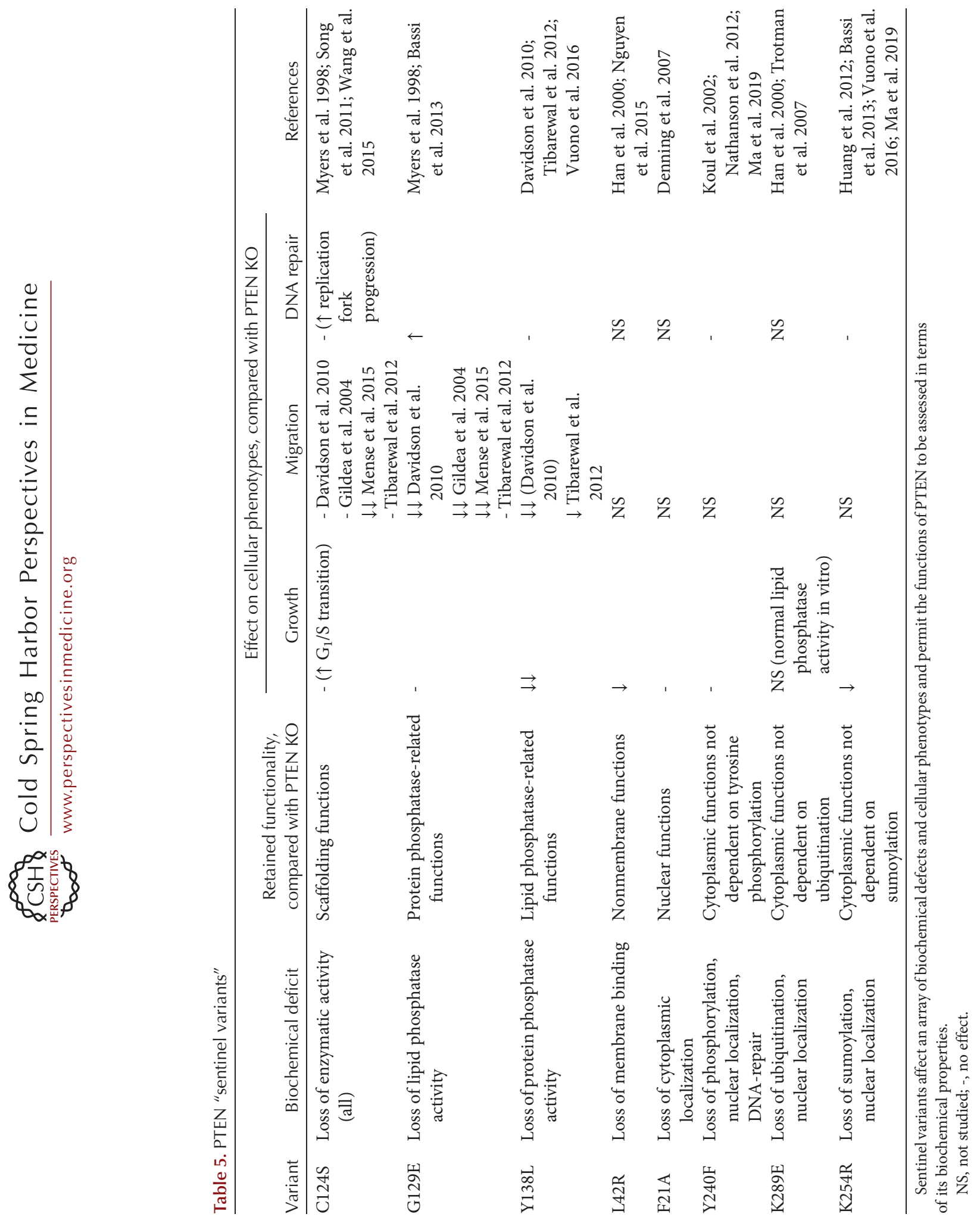
N. Hasle et al.

they only examine two aspects of PTEN biology: steady-state abundance and lipid phosphatase activity. PTEN's protein phosphatase activity and its effects on DNA repair, morphology, and migration may also be clinically relevant. For example, a mere 2\% of the 293 missense PTEN missense variants in the MSK-IMPACT study, which sequenced a diverse array of cancer types, have been characterized according to their effect on DNA repair (Schrader et al. 2016). Given that DNA damaging agents are standard of care for many PTEN-mutant tumors, a MAVE focused on DNA repair could be of clinical use (Lin et al. 2014). MAVEs for invasion and neuronal morphology could be similarly useful for predicting metastasis (Gildea et al. 2004; Bucheit et al. 2014; Cai et al. 2015) and autism spectrum disorder (Haws et al. 2014; Spinelli et al. 2015), respectively.

\section{CONCLUDING REMARKS}

Missense variants have been critical tools for understanding the complex relationships between PTEN's amino acid sequence, molecular functions, and cellular effects. Here, we identify eight variants that offered key insights into PTEN by altering molecular functions in unique, defined ways (Table 5). Three of these variants (Y138L, G129E, and C124S) abrogate protein phosphatase activity, lipid phosphatase activity, or both activities. These enzyme activity separation-of-function variants have been shown to impact multiple cellular phenotypes, which can occur in a context dependent manner, highlighting the complexity of PTEN function. The remaining five variants appear to affect constitutive subcellular localization (L42R, F21A) or posttranslational modification (K254R, K289E, and Y240F). In contrast to the enzyme activity variants, the mechanism by which these variants affect migration and DNA repair has not been studied in detail; understanding their effects may shed light on how PTEN affects these critical cellular processes.

When studied together, separation of function variants allowed experimentalists to determine how cellular phenotypes correlate with particular PTEN enzymatic activities, subcellu- lar distributions, or posttranslational modifications. For example, much of PTEN's role in DNA repair requires both protein phosphatase activity and nuclear localization, suggesting that PTEN's dephosphorylation of nuclear protein substrates is mechanistically critical. Use of these variants is not without caveats, however; insights based on these variants assume that each variant is deficient for a defined set of molecular functions, but none have been exhaustively characterized. Furthermore, as new PTEN molecular functions are characterized, this list will undoubtedly need to be updated.

Biochemical characterization of clinically ascertained PTEN variants have been critical for our understanding of PTEN's molecular functions and cellular phenotypic effects. As such, further characterization of disease associated R130 and R173 variants may yield new insights into PTEN's role in disease. Looking forward, the prevalence of missense variants in sequencing databases will be a valuable source of information to combine with high-throughput variant characterization. By expanding the repertoire of PTEN MAVEs, dissection of PTEN along its many axes of function will become possible. In turn, these datasets will hopefully resolve many variants of unknown significance, aid cancer risk predictions in patients with germline PTEN variants and help provide cancer patients with accurate prognoses and efficacious therapies.

\section{ACKNOWLEDGMENTS}

This work was supported by the National Institutes of Health (NIH) Grants R01GM109110 (D.M.F.), F30CA236335-01 (N.H.) and the American Cancer Society Grant PF-15-221-01 (K.A.M.). D.M.F. is a CIFAR Azrieli Global Scholar.

\section{REFERENCES}

Al-Khouri AM, Ma Y, Togo SH, Williams S, Mustelin T. 2005. Cooperative phosphorylation of the tumor suppressor phosphatase and tensin homologue (PTEN) by casein kinases and glycogen synthase kinase $3 \beta$. J Biol Chem 280: 35195-35202. doi:10.1074/jbc.M503045200 
Andrés-Pons A, Rodríguez-Escudero I, Gil A, Blanco A, Vega A, Molina M, Pulido R, Cid VJ. 2007. In vivo functional analysis of the counterbalance of hyperactive phosphatidylinositol 3-kinase p110 catalytic oncoproteins by the tumor suppressor PTEN. Cancer Res 67: 9731-9739. doi:10.1158/0008-5472.CAN-07-1278

Bassi C, Ho J, Srikumar T, Dowling RJO, Gorrini C, Miller SJ, Mak TW, Neel BG, Raught B, Stambolic V. 2013. Nuclear PTEN controls DNA repair and sensitivity to genotoxic stress. Science 341: 395-399. doi:10.1126/science .1236188

Bucheit AD, Chen G, Siroy A, Tetzlaff M, Broaddus R, Milton D, Fox P, Bassett R, Hwu P, Gershenwald JE, et al 2014. Complete loss of PTEN protein expression correlates with shorter time to brain metastasis and survival in stage IIIB/C melanoma patients with BRAFV600 mutations. Clin Cancer Res 20: 5527-5536. doi:10.1158/10780432.CCR-14-1027

Butler MG, Dazouki MJ, Zhou XP, Talebizadeh Z, Brown M, Takahashi TN, Miles JH, Wang CH, Stratton R, Pilarski R, et al. 2005. Subset of individuals with autism spectrum disorders and extreme macrocephaly associated with germline PTEN tumour suppressor gene mutations. $J$ Med Genet 42: 318-321. doi:10.1136/jmg.2004.024646

Cai LM, Lyu XM, Luo WR, Cui XF, Ye YF, Yuan CC, Peng QX, Wu DH, Liu TF, Wang E, et al. 2015. EBV-miR$B A R T 7-3 p$ promotes the EMT and metastasis of nasopharyngeal carcinoma cells by suppressing the tumor suppressor PTEN. Oncogene 34: 2156-2166. doi:10.1038/ onc.2014.341

Cerami E, Gao J, Dogrusoz U, Gross BE, Sumer SO, Aksoy BA, Jacobsen A, Byrne CJ, Heuer ML, Larsson E, et al. 2012. The cBio cancer genomics portal: An open platform for exploring multidimensional cancer genomics data. Cancer Discov 2: 401-404. doi:10.1158/2159-8290.CD12-0095

Das S, Dixon JE, Cho W. 2003. Membrane-binding and activation mechanism of PTEN. Proc Natl Acad Sci 100: 7491-7496. doi:10.1073/pnas.0932835100

Davidson L, MacCario H, Perera NM, Yang X, Spinelli L, Tibarewal P, Glancy B, Gray A, Weijer CJ, Downes CP, et al. 2010. Suppression of cellular proliferation and invasion by the concerted lipid and protein phosphatase activities of PTEN. Oncogene 29: 687-697. doi:10.1038/onc .2009 .384

Denning G, Jean-Joseph B, Prince C, Durden DL, Vogt PK. 2007. A short N-terminal sequence of PTEN controls cytoplasmic localization and is required for suppression of cell growth. Oncogene 26: 3930-3940. doi:10.1038/sj .onc. 1210175

Gasperini M, Starita L, Shendure J. 2016. The power of multiplexed functional analysis of genetic variants. Nat Protoc 11: 1782-1787. doi:10.1038/nprot.2016.135

Gildea JJ, Herlevsen M, Harding MA, Gulding KM, Moskaluk CA, Frierson HF, Theodorescu D. 2004. PTEN can inhibit in vitro organotypic and in vivo orthotopic invasion of human bladder cancer cells even in the absence of its lipid phosphatase activity. Oncogene 23: 6788-6797. doi:10.1038/sj.onc.1207599

Gu T, Zhang Z, Wang J, Guo J, Shen WH, Yin Y. 2011. CREB is a novel nuclear target of PTEN phosphatase. Cancer Res 71: 2821-2825. doi:10.1158/0008-5472.CAN-10-3399
Han S-Y, Kato H, Kato S, Suzuki T, Shibata H, Ishii S, Shiiba K-I, Matsuno S, Kanamaru R, Ishioka C. 2000. Functional evaluation of PTEN missense mutations using in vitro phosphoinositide phosphatase assay. Cancer Res 60: 3147-3151.

Haws ME, Jaramillo TC, Espinosa F, Widman AJ, Stuber GD, Sparta DR, Tye KM, Russo SJ, Parada LF, Stavarache $\mathrm{M}$, et al. 2014. PTEN knockdown alters dendritic spine/ protrusion morphology, not density. J Comp Neurol 522: 1171-1190. doi:10.1002/cne.23488

He J, Zhang Z, Ouyang M, Yang F, Hao H, Lamb KL, Yang J, Yin Y, Shen WH. 2016. PTEN regulates EG5 to control spindle architecture and chromosome congression during mitosis. Nat Commun 7: 12355. doi:10.1038/ ncomms 12355

Hu Y, Xu S, Jin W, Yi Q, Wei W. 2014. Effect of the PTEN gene on adhesion, invasion and metastasis of osteosarcoma cells. Oncol Rep 32: 1741-1747. doi:10.3892/or.2014 .3362

Huang J, Yan J, Zhang J, Zhu S, Wang Y, Shi T, Zhu C, Chen C, Liu X, Cheng J, et al. 2012. SUMO1 modification of PTEN regulates tumorigenesis by controlling its association with the plasma membrane. Nat Commun 3: 911912. doi:10.1038/ncomms 1919

Ie Kim H, Lee ST. 2009. Oncogenic functions of PTK6 are enhanced by its targeting to plasma membrane but abolished by its targeting to nucleus. J Biochem 146: 133-139. doi:10.1093/jb/mvp050

Koul D, Jasser SA, Lu Y, Davies MA, Shen R, Shi Y, Yung WKA. 2002. Motif analysis of the tumor suppressor gene MMAC/PTEN identifies tyrosines critical for tumor suppression and lipid phosphatase activity. Oncogene 21: 2357-2364. doi:10.1038/sj.onc.1205296

Landrum MJ, Lee JM, Benson M, Brown GR, Chao C, Chitipiralla S, Gu B, Hart J, Hoffman D, Jang W, et al. 2018. ClinVar: Improving access to variant interpretations and supporting evidence. Nucleic Acids Res 46: D1062D1067. doi:10.1093/nar/gkx1153

Lee JO, Yang H, Georgescu MM, Di Cristofano A, Maehama T, Shi Y, Dixon JE, Pandolfi P, Pavletich NP. 1999. Crystal structure of the PTEN tumor suppressor: Implications for its phosphoinositide phosphatase activity and membrane association. Cell 99: 323-334. doi:10.1016/S0092-8674 (00)81663-3

Leslie NR, Longy M. 2016. Inherited PTEN mutations and the prediction of phenotype. Semin Cell Dev Biol 52: 3038. doi:10.1016/j.semcdb.2016.01.030

Li J, Yen C, Liaw D, Podsypanina K, Bose S, Wang SI, Puc J, Miliaresis C, Rodgers L, McCombie R, et al. 1997. PTEN, a putative protein tyrosine phosphatase gene mutated in human brain, breast, and prostate cancer. Science 275: 1943-1948.

Liaw D, Marsh DJ, Li J, Dahia PLM, Wang SI, Zheng Z, Bose S, Call KM, Tsou HC, Peacocke M, et al. 1997. Germline mutations of the PTEN gene in Cowden disease, an inherited breast and thyroid cancer syndrome. Nat Genet 16: 64-67. doi:10.1038/ng0597-64

Lin F, De Gooijer MC, Roig EM, Buil LCM, Christner SM, Beumer JH, Wurdinger T, Beijnen JH, Van Tellingen O. 2014. ABCB1, ABCG2, and PTEN determine the response of glioblastoma to temozolomide and ABT-888 
N. Hasle et al.

therapy. Clin Cancer Res 20: 2703-2713. doi:10.1158/ 1078-0432.CCR-14-0084

Ma J, Benitez JA, Li J, Kolodner RD, Chen CC, Furnari FB, Ma J, Benitez JA, Li J, Miki S, et al. 2019. Inhibition of nuclear PTEN tyrosine phosphorylation enhances glioma radiation sensitivity through attenuated DNA repair Cancer Cell 35: 504-518.e7. doi:10.1016/j.ccell.2019.01 .020

Marsh DJ, Kum JB, Lunetta KL, Bennett MJ, Gorlin RJ, Ahmed SF, Bodurtha J, Crowe C, Curtis MA, Dasouki $\mathrm{M}$, et al. 1999. PTEN mutation spectrum and genotypephenotype correlations in Bannayan-Riley-Ruvalcaba syndrome suggest a single entity with Cowden syndrome. Hum Mol Genet 8: 1461-1472. doi:10.1093/hmg/8.8.1461

Matreyek KA, Starita LM, Stephany JJ, Martin B, Chiasson MA, Gray VE, Kircher M, Khechaduri A, Dines JN, Hause RJ, et al. 2018. Multiplex assessment of protein variant abundance by massively parallel sequencing. Nat Genet 50: 874-882. doi:10.1038/s41588-018-0122-Z

Mense SM, Barrows D, Hodakoski C, Steinbach N, Schoenfeld D, Su W, Hopkins BD, Su T, Fine B, Hibshoosh H, et al. 2015. PTEN inhibits PREX2-catalyzed activation of RAC1 to restrain tumor cell invasion. Sci Signal 8: ra32. doi:10.1126/scisignal.2005840

Mester JL, Ghosh R, Pesaran T, Huether R, Karam R, Hruska KS, Costa HA, Lachlan K, Ngeow J, Barnholtz-Sloan J, et al. 2018. Gene-specific criteria for PTEN variant curation: Recommendations from the ClinGen PTEN expert panel. Hum Mutat 39: 1581-1592. doi:10.1002/humu.23636

Mighell TL, Evans-Dutson S, O’Roak BJ. 2018. A saturation mutagenesis approach to understanding PTEN lipid phosphatase activity and genotype-phenotype relationships. Am J Hum Genet 102: 943-955. doi:10.1016/j .ajhg.2018.03.018

Myers MP, Stolarov JP, Eng C, Li J, Wang SI, Wigler MH, Parsons R, Tonks NK. 1997. P-TEN, the tumor suppressor from human chromosome $10 \mathrm{q} 23$, is a dual-specificity phosphatase. Proc Natl Acad Sci 94: 9052-9057. doi:10 $.1073 /$ pnas.94.17.9052

Myers MP, Pass I, Batty IH, Van Der Kaay J, Stolarov JP, Hemmings BA, Wigler MH, Downes CP, Tonks NK 1998. The lipid phosphatase activity of PTEN is critical for its tumor supressor function. Proc Natl Acad Sci 95: 13513-13518. doi:10.1073/pnas.95.23.13513

Nathanson D, Bachoo RM, Wykosky J, Fenton TR, Furnari FB, Mischel PS, Dang J, de Albuquerque C P, Tanaka K, Vandenberg SR, et al. 2012. Resistance to EGF receptor inhibitors in glioblastoma mediated by phosphorylation of the PTEN tumor suppressor at tyrosine 240 Proc Natl Acad Sci 109: 14164-14169. doi:10.1073/pnas .1211962109

Nguyen HN, Yang JM, Afkari Y, Park BH, Sesaki H, Devreotes PN, Iijima M. 2014a. Engineering ePTEN, an enhanced PTEN with increased tumor suppressor activities. Proc Natl Acad Sci 111: E2684-E2693. doi:10.1073/pnas 1409433111

Nguyen HN, Afkari Y, Senoo H, Sesaki H, Devreotes PN, Iijima M. 2014b. Mechanism of human PTEN localization revealed by heterologous expression in Dictyostelium. Oncogene 33: 5688-5696. doi:10.1038/onc.2013 .507
Nguyen HN, Yang JM Jr, Rahdar M, Keniry M, Swaney KF, Parsons R, Park BH, Sesaki H, Devreotes PN, Iijima M. 2015. A new class of cancer-associated PTEN mutations defined by membrane translocation defects. Oncogene 34: 3737-3743. doi:10.1038/onc.2014.293

Odriozola L, Singh G, Hoang T, Chan AM. 2007. Regulation of PTEN activity by its carboxyl-terminal autoinhibitory domain. J Biol Chem 282: 23306-23315. doi:10.1074/jbc .M611240200

Papa A, Wan L, Bonora M, Salmena L, Song MS, Hobbs RM, Lunardi A, Webster K, Ng C, Newton RH, et al. 2014. Cancer-associated PTEN mutants act in a dominant-negative manner to suppress PTEN protein function. Cell 157: 595-610. doi:10.1016/j.cell.2014.03.027

Raftopoulou M, Etienne-Manneville S, Self A, Nicholls S, Hall A. 2004. Regulation of cell migration by the C2 domain of the tumor suppressor PTEN. Science 303: 11791181. doi:10.1126/science.1092089

Rahdar M, Inoue T, Meyer T, Zhang J, Vazquez F, Devreotes PN. 2009. A phosphorylation-dependent intramolecular interaction regulates the membrane association and activity of the tumor suppressor PTEN. Proc Natl Acad Sci 106: 480-485. doi:10.1073/pnas.0811212106

Richards S, Aziz N, Bale S, Bick D, Das S, Gastier-Foster J, Grody WW, Hegde M, Lyon E, Spector E, et al. 2015. Standards and guidelines for the interpretation of sequence variants: A joint consensus recommendation of the American College of Medical Genetics and Genomics and the Association for Molecular Pathology. Genet Med 17: 405-423. doi:10.1038/gim.2015.30

Rodríguez-Escudero I, Roelants FM, Thorner J, Nombela C, Molina M, Cid VJ. 2005. Reconstitution of the mammalian PI3K/PTEN/Akt pathway in yeast. Biochem J 390: 613-623. doi:10.1042/BJ20050574

Rodríguez-Escudero I, Oliver MD, Andrés-Pons A, Molina M, Cid VJ, Pulido R. 2011. A comprehensive functional analysis of PTEN mutations: Implications in tumor- and autism-related syndromes. Hum Mol Genet 20: 41324142. doi:10.1093/hmg/ddr337

Schaller MD, Borgman CA, Cobb BS, Vines RR, Reynolds AB, Parsons JT. 1992. pp125FAK, a structurally distinctive protein-tyrosine kinase associated with focal adhesions. Proc Natl Acad Sci 89: 5192-5196. doi:10.1073/ pnas.89.11.5192

Schrader KA, Cheng DT, Joseph V, Prasad M, Walsh M, Zehir A, Ni A, Thomas T, Benayed R, Ashraf A, et al. 2016. Germline variants in targeted tumor sequencing using matched normal DNA. JAMA Oncol 2: 104-111. doi:10.1001/jamaoncol.2015.5208

Smith IN, Thacker S, Seyfi M, Cheng F, Eng C. 2019. Conformational dynamics and allosteric regulation landscapes of germline PTEN mutations associated with autism compared to those associated with cancer. Am J Hum Genet 104: 861-878. doi:10.1016/j.ajhg.2019 .03 .009

Song MS, Carracedo A, Salmena L, Song SJ, Egia A, Malumbres M, Pandolfi PP. 2011. Nuclear PTEN regulates the APC-CDH1 tumor-suppressive complex in a phosphatase-independent manner. Cell 144: 187-199. doi:10 $.1016 /$ j.cell.2010.12.020

Spinelli L, Black FM, Berg JN, Eickholt BJ, Leslie NR. 2015. Functionally distinct groups of inherited PTEN mutations 
in autism and tumour syndromes. J Med Genet 52: 128134. doi:10.1136/jmedgenet-2014-102803

Stambolic V, Suzuki A, Dela Pompa JL, Brothers GM, Mirtsos C, Sasaki T, Ruland J, Penninger JM, Siderovski DP, Mak TW. 1998. Negative regulation of PKB/Akt-dependent cell survival by the tumor suppressor PTEN. Cell 95: 29-39.

Starita LM, Ahituv N, Dunham MJ, Kitzman JO, Roth FP, Seelig G, Shendure J, Fowler DM. 2017. Variant interpretation: Functional assays to the rescue. Am J Hum Genet 101: 315-325. doi:10.1016/j.ajhg.2017.07.014

Tamura M, Gu J, Matsumoto K, Aota S, Parsons RE, Yamada KM. 1998. Inhibition of cell migration, spreading, and focal adhesions by tumor suppressor PTEN. Science 280: 1614-1617.

Tibarewal P, Zilidis G, Spinelli L, Schurch N, Maccario H, Gray A, Perera NM, Davidson L, Geoffrey J, Leslie NR, et al. 2012. PTEN protein phosphatase activity correlates with control of gene expression and invasion, a tumorsuppressing phenotype, but not with AKT activity. Sci Signal 5: ra18. doi:10.1126/scisignal.2002138

Torres J, Pulido R. 2001. The tumor suppressor PTEN is phosphorylated by the protein kinase CK2 at its C terminus. Implications for PTEN stability to proteasome-mediated degradation. J Biol Chem 276: 993-998. doi:10 $.1074 /$ jbc.M009134200

Trotman LC, Wang X, Alimonti A, Chen Z, Teruya-Feldstein J, Yang H, Pavletich NP, Carver BS, Cordon-Cardo C, Erdjument-Bromage H, et al. 2007. Ubiquitination regulates PTEN nuclear import and tumor suppression. Cell 128: 141-156. doi:10.1016/j.cell.2006.11.040

Vazquez F, Ramaswamy S, Nakamura N, Sellers WR. 2000. Phosphorylation of the PTEN tail regulates protein stability and function. Mol Cell Biol 20: 5010-5018. doi:10 $.1128 /$ MCB.20.14.5010-5018.2000
Vazquez F, Matsuoka S, Sellers WR, Yanagida T, Ueda M, Devreotes PN. 2006. Tumor suppressor PTEN acts through dynamic interaction with the plasma membrane Proc Natl Acad Sci 103: 3633-3638. doi:10.1073/pnas .0510570103

Vuono EA, Mukherjee A, Vierra DA, Adroved MM, Hodson C, Deans AJ, Howlett NG. 2016. The PTEN phosphatase functions cooperatively with the Fanconi anemia proteins in DNA crosslink repair. Sci Rep 6: 1-13. doi:10.1038/ srep36439

Wang X, Trotman LC, Koppie T, Alimonti A, Chen Z, Gao Z, Wang J, Erdjument-Bromage H, Tempst P, CordonCardo C, et al. 2007. NEDD4-1 is a proto-oncogenic ubiquitin ligase for PTEN. Cell 128: 129-139. doi:10 $.1016 /$ j.cell.2006.11.039

Wang G, Li Y, Wang P, Liang H, Cui M, Zhu M, Guo L, Su Q, Sun Y, McNutt MA, et al. 2015. PTEN regulates RPA1 and protects DNA replication forks. Cell Res 25: 1189-1204. doi:10.1038/cr.2015.115

Wozniak DJ, Kajdacsy-Balla A, Macias V, Ball-Kell S, Zenner ML, Bie W, Tyner AL. 2017. PTEN is a protein phosphatase that targets active PTK6 and inhibits PTK6 oncogenic signaling in prostate cancer. Nat Commun 8: 1508 . doi:10.1038/s41467-017-01574-5

Yehia L, Ngeow J, Eng C. 2019. PTEN-opathies: From biological insights to evidence-based precision medicine. J Clin Invest 129: 452-464. doi:10.1172/JCI121277

Zhang XC, Piccini A, Myers MP, Van Aelst L, Tonks NK. 2012. Functional analysis of the protein phosphatase activity of PTEN. Biochem J 444: 457-464.

Zhang LL, Liu J, Lei S, Zhang J, Zhou W, Yu HG. 2014. PTEN inhibits the invasion and metastasis of gastric cancer via downregulation of FAK expression. Cell Signal 26: 10111020. doi:10.1016/j.cellsig.2014.01.025 


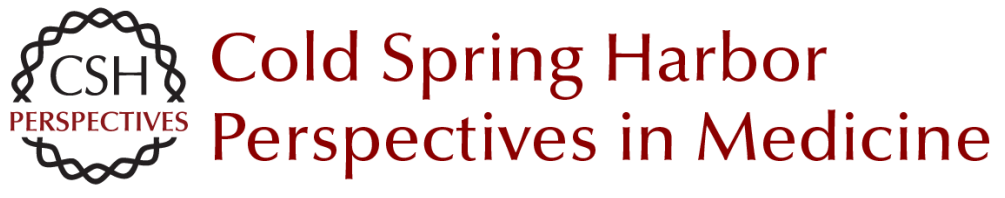

\section{The Impact of Genetic Variants on PTEN Molecular Functions and Cellular Phenotypes}

Nicholas Hasle, Kenneth A. Matreyek and Douglas M. Fowler

Cold Spring Harb Perspect Med 2019; doi: 10.1101/cshperspect.a036228 originally published online August 26, 2019

\section{Subject Collection The PTEN Family}

\section{PTEN in Regulating Hematopoiesis and Leukemogenesis Yilin Wu, Haichuan Zhu and Hong Wu}

Connecting Genotype with Behavioral Phenotype in Mouse Models of Autism Associated with PTEN Mutations Amy E. Clipperton-Allen and Damon T. Page

Metabolic Role of PTEN in Insulin Signaling and Resistance

$$
\text { Yu Zhe Li, Antonio Di Cristofano and Minna Woo }
$$

Posttranslational Regulation and Conformational

Plasticity of PTEN

Larissa Kotelevets, Barbara Trifault, Eric Chastre, et al.

Toward Systems Pathology for PTEN Diagnostics Nahal Haddadi, Glena Travis, Najah T. Nassif, et al.

PTEN in Hereditary and Sporadic Cancer Joanne Ngeow and Charis Eng

PTEN Mouse Models of Cancer Initiation and Progression

Yu-Ru Lee and Pier Paolo Pandolfi

Dual-Specific Protein and Lipid Phosphatase

PTEN and Its Biological Functions

Taojian Tu, Jingyu Chen, Lulu Chen, et al.
PTEN: Bridging Endocytosis and Signaling Matthew F. Lee and Lloyd C. Trotman

PTEN as a Guardian of the Genome: Pathways and Targets

Xinyi Fan, Jeffrey Kraynak, Jonathan P.S. Knisely, et al.

Discovery of the PTEN Tumor Suppressor and Its

Connection to the PI3K and AKT Oncogenes Ramon Parsons

The Complex Landscape of PTEN mRNA

Regulation Erin Sellars, Martino Gabra and Leonardo Salmena

PTEN Nuclear Functions Jason Ho, Edward S. Cruise, Ryan J.O. Dowling, et al.

Structural Mechanisms of PTEN Regulation Glenn R. Masson and Roger L. Williams

PTEN in Chromatin Remodeling Jingyi Yang and Yuxin Yin

The Role of PTEN in Innate and Adaptive Immunity Henry Taylor, Arian D. J. Laurence and Holm H. Uhlig

For additional articles in this collection, see http://perspectivesinmedicine.cshlp.org/cgi/collection/ 